\title{
Mass Flow Rate Measurement of Thermal Creep Flow from Transitional to Slip Flow Regime
}

\author{
Hiroki Yamaguchi ${ }^{1} \dagger$, Pierre Perrier ${ }^{2}$, Minh Tuan $\mathbf{H o}^{2,3}$, J. Gilbert \\ Méolans $^{2}$, Tomohide Niimi ${ }^{1}$, and Irina Graur ${ }^{2}$ \\ ${ }^{1}$ Dept. of Micro-Nano Systems Engineering, Nagoya University, \\ Furo-cho, Chikusa, Nagoya, Aichi, 464-8603 Japan \\ 2 Aix-Marseille Université, CNRS, IUSTI UMR 7343, \\ 5 rue Enrico Fermi, 13013 Marseille, France \\ 3 James Weir Fluids Laboratory, Department of Mechanical and Aerospace Engineering, \\ University of Strathclyde, \\ James Weir Building, 75 Montrose Street, Glasgow G1 1XJ, UK
}

(Received ?; revised ?; accepted ?.)

The measurements of the thermal creep flow through a single rectangular micro channel connected to two tanks maintained initially at the same pressure, but at different temperatures, is carried out for five noble gas species, in a large pressure variation range and for two temperature differences between the tanks. The time dependent pressure variations in both cold and hot tanks are investigated, and the temperature driven (thermal creep) mass flow rate between two tanks is calculated from these data for the rarefaction parameter ranging from the transitional to slip flow regime. The measured mass flow rate is compared with the numerical solution of the S-model kinetic equation, showing good agreement with each other. A novel approximative expression to calculate the temperature driven mass flow rate in the transitional and slip flow regimes is proposed. This expression provides the results in good agreement with the measured values of the mass flow rate. In the slip flow regime, the thermal slip coefficient is calculated by employing the previously reported methodology, and the influence of the gas nature on this coefficient is investigated. The measured values of the thermal slip coefficient agree well with the values available in the literature, indicating that this coefficient is independent of the shape of a channel.

\section{Introduction}

The thermal creep flow is one of the most interesting phenomena in the relatively high Knudsen number flows. This flow is induced by a temperature gradient along a surface, and it is directed from the cold to hot side. The Knudsen number, defined by the ratio of the molecular mean free path $\lambda$ to a characteristic length of a system $\mathcal{L}$ as $K n=\lambda / \mathcal{L}$, becomes large at a low pressure environment and/or in a small-scale flow field. Recently, it draws much attention due to the rapid development of the micro- and nano-technologies (Vargo et al. 1999; Passian et al. 2003; Young et al. 2003; Alexeenko et al. 2006; Han et al. 2007; Gupta \& Gianchandani 2008; Sharipov 2011; Gupta et al. 2012; Martini et al. 2012; Dongari \& Agrawal 2012; Graur \& Ho 2014; Yamaguchi et al. 2014; Rojas-Cárdenas et al. 2015).

To analyze such thermal-flow field in the slip flow regime, the Navier-Stokes equations 
with the velocity slip boundary conditions are often employed (Kogan 1969; Cercignani 1990; Sharipov 2011). Two coefficients are usually involved in the expression of this velocity slip condition: the velocity slip and thermal slip coefficients. Among them, the thermal slip coefficient plays an important role in the thermal creep flow; therefore, we focus the present study on this coefficient.

The values of the thermal slip coefficient were derived theoretically from the kinetic theory of gases, using various kinetic equations (Boltzmann equation and model kinetic equations) and different models of the intermolecular potential, see review in Sharipov (2011). However, it is very important to improve our knowledge on the thermal slip coefficient and to obtain experimentally its values, especially because up to now only three works were devoted to the experimental extraction of this coefficient (Annis 1972; Porodnov et al. 1978; Rojas-Cárdenas et al. 2015). First, the thermal slip coefficient was extracted from the measurements of the thermomolecular pressure difference induced by the flow through a circular capillary, submitted to the large and small temperature gradient conditions in Annis (1972) and Porodnov et al. (1978), respectively. Recently, a novel procedure of the thermal slip coefficient extraction was proposed (Rojas-Cárdenas et al. 2015). The authors measured the stationary temperature driven mass flow rate through a micro tube of the circular cross-section to obtain then this coefficient. It is clear that with only these few experimental results, the information on the temperature driven flows and also on the thermal slip coefficient is not complete enough. Therefore, the new experimental data on the temperature driven gas mass flow rate are indispensable not only for the thermal management of the micro devices with the temperature gradients, but also for the extraction of the thermal slip coefficients for various gases.

In this study, we used our previously obtained (Yamaguchi et al. 2014) experimental data on pressure variation in time for the temperature driven gas flow through a rectangular micro channel to obtain new reliable characteristics on the thermal creep flow. The experimental values of the thermal slip coefficient are provided for five noble gases (Helium, Neon, Argon, Krypton, Xenon) and the influence of the gas nature on this coefficient is studied. Moreover, a novel procedure to obtain the temperature driven mass flow rate in the transitional flow regime, useful in many rarefied flows applications, is proposed. The experimental data are analyzed to characterize the thermal creep flows in wide flow regimes ranging from the transitional to slip flow regime.

\section{Experimental data analysis}

The details and sketch of the experimental apparatus used in present work can be found in Yamaguchi et al. (2014). The rectangular micro channel made of PEEK with

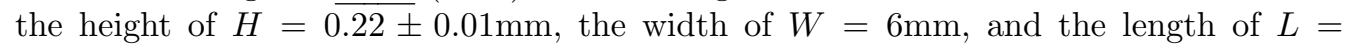
$73 \mathrm{~mm}$ was employed. The temperature gradient along the micro channel was realized by two blocks with gas reservoirs inside placed at both ends of the micro channel, whose temperatures were controlled by an electrical heater for the hot side and a circulating water for the cold side, respectively. The temperature of each block was monitored by a K-type thermocouple (TC) placed on the outside of the blocks. Temperature for the hot reservoir $T_{\mathrm{H}}$ and that for the cold reservoir $T_{\mathrm{C}}$ were maintained to realize two cases of the temperature difference $\Delta T: 1) T_{\mathrm{H}}=347.1 \pm 0.5 \mathrm{~K}, T_{\mathrm{C}}=289.2 \pm 0.2 \mathrm{~K}$, where $\Delta T=57.9 \mathrm{~K}$ and 2) $T_{\mathrm{H}}=337.0 \pm 0.6 \mathrm{~K}, T_{\mathrm{C}}=299.6 \pm 0.4 \mathrm{~K}$, where $\Delta T=37.4 \mathrm{~K}$. The both temperature differences were chosen to have the same mean temperature $T_{\mathrm{m}}=\left(T_{\mathrm{C}}+T_{\mathrm{H}}\right) / 2$ for two cases as $T_{\mathrm{m}}=318 \mathrm{~K}$. Here, twice of the standard deviation $(2 \sigma)$ was used as an uncertainty of the temperature conditions.

The hot and cold reservoirs were connected directly by a tube with a large diameter 
forming a circuit with insertion of a micro valve (Parker Hannifin). When the micro valve is open, there is the stationary thermal creep flow through the micro channel from the cold to hot sides; while the flow through the large diameter tube maintains pressures equally at both reservoirs by compensating the thermal creep. By closing the micro valve, the flow in the large diameter tube is blocked, and there is only the thermal creep flow inside the micro channel. With time evolution, the pressure difference occurs between the cold and hot reservoirs as a result of the thermal creep flow, inducing a pressure driven flow in the opposite direction to the thermal creep flow. The pressure variations with time in two reservoirs are well fitted by the exponential functions (Yamaguchi et al. 2014; Rojas-Cárdenas et al. 2011, 2013) as,

$$
\begin{aligned}
& p_{\mathrm{C}}(t)=p_{\mathrm{i}}-\left|p_{\mathrm{C}, \infty}-p_{\mathrm{i}}\right|\left(1-e^{-t / \tau_{\mathrm{C}}}\right), \\
& p_{\mathrm{H}}(t)=p_{\mathrm{i}}+\left|p_{\mathrm{H}, \infty}-p_{\mathrm{i}}\right|\left(1-e^{-t / \tau_{\mathrm{H}}}\right),
\end{aligned}
$$

where $p_{\mathrm{C}}(t)$ and $p_{\mathrm{H}}(t)$ are the pressure variation in time in the cold and hot reservoirs, respectively, $t$ is a time, $p_{\mathrm{i}}$ is the initial pressure, which was common for the hot and cold reservoirs, $p_{\mathrm{C}, \infty}$ and $p_{\mathrm{H}, \infty}$ are the final equilibrium pressures in the cold and hot reservoirs, respectively, and $\tau_{\mathrm{C}}$ and $\tau_{\mathrm{H}}$ are the relaxation times, at the cold and hot parts, respectively. The pressures in two reservoirs were measured by capacitance diaphragm gauges (CDGs) (Inficon CDG045D (hot side), CDG025 (cold side)); but there were temperature differences between reservoirs and CDGs, resulting, in the both cold and hot sides, in pressure differences between a reservoir and a CDG due to the thermal creep flow along the CDG tube. Therefore, the pressure was corrected by using the Takaishi \& Sensui formula (Takaishi \& Sensui 1963).

The time $t=0$ was determined as the closing moment of the micro valve, which was decided by monitoring an applied voltage to the micro valve. Since the stationary thermal creep flow was not yet disturbed at $t=0$, the mass flow rate of the thermal creep flow is obtained from the measured time variation of pressures as,

$$
\dot{M}=\left.\frac{V}{R T} \frac{d p(t)}{d t}\right|_{t=0},
$$

where $V, T, p(t)$ are the volume, the temperature, and pressure of a reservoir, respectively, and $R$ is the specific gas constant. The pressure variation rate $d p /\left.d t\right|_{t=0}$ was calculated by differentiating the fitted exponential functions (Eq. (2.1)). The stationary mass flow rate can be calculated for each of the cold and hot reservoirs, which should be equal from the mass conservation. In this study, the mass flow rate of the cold reservoir, calculated from $p_{\mathrm{C}}(t)$, is used, because there is a slight delay at the starting of the pressure variation in the hot reservoir. The reason of the delay might be the fact that the temperature difference was quite large between the hot reservoir $\left(74^{\circ} \mathrm{C}\right.$ or $\left.64^{\circ} \mathrm{C}\right)$ and the internal temperature of the pressure gauge $\left(45^{\circ} \mathrm{C}\right)$, where a thermal creep flow was induced and it could affect the pressure measurement.

To analyze the time dependent characteristics of the thermal creep flow, namely the mass flow rate, the rarefaction parameter is used

$$
\delta=\frac{p H}{\mu \sqrt{2 R T}},
$$

which is inversely proportional to the Knudsen number. In this study, the channel height $H$ is chosen as the characteristic length of the system. The viscosity coefficient $\mu$ is 
assumed to have the power law dependence on temperature,

$$
\mu=\mu_{\mathrm{ref}}\left(\frac{T}{T_{\mathrm{ref}}}\right)^{\omega},
$$

where $\mu_{\text {ref }}$ is the viscosity coefficient at the temperature $T_{\text {ref }}$ and $\omega$ is the viscosity index (Bird 1994). The rarefaction parameter is defined by Eq. (2.3) as a local value, depending on the local values of pressure and temperature, which were, however, not measured. Therefore, the mean rarefaction parameter $\delta_{\mathrm{m}}$ was calculated by using the mean temperature $T_{\mathrm{m}}$ and the initial pressure $p_{\mathrm{i}}$ as,

$$
\delta_{\mathrm{m}}=\frac{p_{\mathrm{i}} H}{\mu\left(T_{\mathrm{m}}\right) \sqrt{2 R T_{\mathrm{m}}}} .
$$

The initial pressure $p_{\mathrm{i}}$ was varied from $66 \mathrm{~Pa}$ to $1270 \mathrm{~Pa}$ for every gas species, and the mean rarefaction parameter $\delta_{\mathrm{m}}$ in the range between 0.6 to 40 were employed. Therefore, the flow was ranged from the transitional to slip flow regime.

\section{Numerical simulations to estimate the mass flow rate inside the channel}

\subsection{Numerical mass flow rate}

The detailed information about the local flow characteristics inside the channel is important for the analysis of the average characteristic measurements, as the mass flow rate; however, it was not easy to obtain them experimentally. Therefore, we also employed a numerical simulation for the further analysis.

Various authors have analyzed stationary thermal creep flows through rectangular cross-section channels or parallel plates by using the linearized kinetic models (BGK (Stvorik et al. 1978) and S-model (Sharipov 1999; Graur \& Ho 2014)) or by solving the linearized Boltzmann equation (Ohwada et al. 1989; Sone 2002). These approaches are indispensables in the transitional flow regime, where the continuum approach does not valid anymore, but the collisionless Boltzmann equation cannot be applied yet. In all these publications, it was assumed that the local pressure and temperature gradients along the channel

$$
\frac{H}{p} \frac{\mathrm{d} p}{\mathrm{~d} z} \ll 1, \quad \frac{H}{T} \frac{\mathrm{d} T}{\mathrm{~d} z} \ll 1,
$$

were small enough for the linearization of the kinetic equations. This assumption, Eq. (3.1), allows the presentation of the mass flow rate through a rectangular cross-section channel in the linear form

$$
\dot{M}=\frac{H W p}{\sqrt{2 R T}}\left(-G_{\mathrm{p}}(\delta, \alpha) \frac{H}{p} \frac{\mathrm{d} p}{\mathrm{~d} z}+G_{\mathrm{T}}(\delta, \alpha) \frac{H}{T} \frac{\mathrm{d} T}{\mathrm{~d} z}\right) .
$$

In expressions (3.1) and (3.2), the $z$-axis coincides with the direction of the temperature gradient and of the channel's largest dimension (channel length $L$ ). The local dimensionless flow rates, $G_{\mathrm{p}}$ and $G_{\mathrm{T}}$, are induced by the local gradients of pressure and temperature, Eqs. (3.1), respectively, and they depend on the local rarefaction parameter $\delta$ and on the accommodation coefficient $\alpha$. In the numerical simulation we assumed, unless otherwise stated, the full accommodation, i.e. $\alpha=1$, at walls in the frame of the Maxwell diffusespecular kernel. It is important to note that $T$ and $p$ in Eq. (3.2) are the temperature and pressure at a local point inside the channel, respectively.

Equation (3.2) was integrated numerically by using the mass conservation property, 
and the values of the dimensionless mass flow rates $G_{\mathrm{p}}(\delta)$ and $G_{\mathrm{T}}(\delta)$, obtained in Graur \& Ho (2014) for the channel with $W / H$ ratio equals to 0.0367 , which corresponds to the real channel's dimensions. The equality of the pressure in both inlet and outlet reservoirs was used as the boundary conditions for the pressure. From this integration, the mass flow rate $\dot{M}$ was obtained together with the pressure distribution along the channel, see Sharipov (1999); Graur \& Ho (2014) for more details. To extract these both flow characteristics, a linear temperature distribution along the channel walls was assumed. In Section 4.1, the numerical results will be compared with the mass flow rate measurements.

\subsection{Effect of pressure profiles inside the micro channel}

The mass flow rate $\dot{M}$, expressed at a local point is, as mentioned above, known to be decomposed into two terms, as in Eq. (3.2). The first and second terms represent the pressure and temperature driven flows, respectively. Even though the flow inside the micro channel at $t=0$ was largely dominated by the temperature driven flow (RojasCárdenas et al. 2013), there would be a slight pressure gradient along the micro channel, which would induce a flow. The fraction of this flow rate, caused by the pressure gradient, can be estimated from the first term of Eq. (3.2), i.e. the reduced flow rate induced by a pressure gradient. To obtain this information on the part of the pressure gradient induced mass flow rate in the total mass flow rate, Eq.(3.2) was integrated along the channel as following,

$$
\begin{aligned}
\dot{M} & =-\frac{1}{L} \int_{p_{\mathrm{C}}}^{p_{\mathrm{H}}} \frac{H^{2} W p}{\sqrt{2 R T}} G_{\mathrm{p}}(\delta) \frac{d p}{p}+\frac{1}{L} \int_{T_{\mathrm{C}}}^{T_{\mathrm{H}}} \frac{H^{2} W p}{\sqrt{2 R T}} G_{\mathrm{T}}(\delta) \frac{d T}{T} \\
& =-\dot{M}_{\mathrm{p}}+\dot{M}_{\mathrm{T}} .
\end{aligned}
$$

To establish Eq. (3.3) from Eq. (3.2), the mass conservation property was used. When integrating the first term in Eq. (3.3), we implemented the pressure distribution along the channel, obtained, as it was explained in Section 3.1, from the numerical solution of Eq.(3.2). For the integration of the second term in Eq. (3.3), the linear distribution of the temperature was assumed. After integration, the ratio of pressure driven mass flow rate, $\dot{M}_{\mathrm{p}}$, to the whole mass flow rate $\dot{M}$ was calculated. It was found that the term $\dot{M}_{\mathrm{p}}$ was appeared to be less than $0.1 \%$ of the full mass flow rate $\dot{M}$ for $\Delta T=37 \mathrm{~K}$ and less than $0.2 \%$ for $\Delta T=58 \mathrm{~K}$, respectively. These results indicate that the pressure profile along the micro channel is nearly flat, i.e. the pressure driven flow is negligible at $t=0$, when the mass flow rate is driven only by the temperature gradient.

\subsection{Temperature driven mass flow rate}

Taking into account the previous results, obtained in Section 3.2, on the part of the pressure induced mass flow rate in the total mass flow rate, it is reasonable to approximate the total mass flow rate, Eq.(3.3), only by the temperature gradient term:

$$
\dot{M} \simeq \dot{M}_{\mathrm{T}}=\frac{1}{L} \int_{T_{\mathrm{C}}}^{T_{\mathrm{H}}} \frac{H^{2} W p}{\sqrt{2 R T}} G_{\mathrm{T}}(\delta) \frac{d T}{T} .
$$

Since this expression depends on local values, it was integrated along the channel to obtain the mean value, which is directly compared with the measured mass flow rate in Section 4.3. Even though $G_{\mathrm{T}}(\delta)$ is a function of the local rarefaction parameter $\delta$, and therefore varies along the channel with $\delta$ varying, $G_{\mathrm{T}}(\delta)$ in the presented here analysis was assumed to be equal to $G_{\mathrm{T}}\left(\delta_{\mathrm{m}}\right)$, because the variation of $\delta$ is not so large along the channel due to the negligible longitudinal pressure variation and the small mean temperature gradient. Also the pressure was assumed to be equal to the initial pressure 
$p_{\mathrm{i}}$ in Eq. (3.5), then, after integration of Eq. (3.5), the thermal creep mass flow rate becomes

$$
\dot{M}_{\mathrm{T}} \simeq \dot{M}_{\mathrm{T}}^{\mathrm{A}}=G_{\mathrm{T}}\left(\delta_{\mathrm{m}}\right) \frac{2 H^{2} W}{L} p_{\mathrm{i}}\left(\frac{1}{\sqrt{2 R T_{\mathrm{C}}}}-\frac{1}{\sqrt{2 R T_{\mathrm{H}}}}\right) .
$$

To validate the two approximations: $G_{\mathrm{T}}(\delta)$ by $G_{\mathrm{T}}\left(\delta_{\mathrm{m}}\right)$ and the local pressure by its initial value $p_{\mathrm{i}}$, when expression (3.6) was obtained, the comparison with the originally simulated mass flow rate $\dot{M}$, deduced from Eq. (3.3), see Section 3, was carried out. The discrepancies $\left(\left(\dot{M}_{\mathrm{T}}^{\mathrm{A}}-\dot{M}\right) / \dot{M}\right)$ appeared to be less than $0.7 \%$ for the whole range of the flow regime, $\delta_{\mathrm{m}}=[0.5,40]$. Thus, it is reasonable to estimate that the variation of $G_{\mathrm{T}}$ as a function of $\delta$ was very small along the channel and that the $G_{\mathrm{T}}(\delta)$ value is well represented by $G_{\mathrm{T}}\left(\delta_{\mathrm{m}}\right)$, as well as to estimate the pressure along the channel by the initial pressure $p_{\mathrm{i}}$. The proposed expression, Eq. (3.6), is directly compared with the measured mass flow rate in Section 4.3.

Expression (3.6) can be also easily modified to show the dependency of the mass flow rate on the gas viscosity

$$
\dot{M}_{\mathrm{T}} \simeq \dot{M}_{\mathrm{T}}^{\mu}=\mu\left(T_{\mathrm{m}}\right) G_{\mathrm{T}}\left(\delta_{\mathrm{m}}\right) \frac{2 H W}{L} \delta_{\mathrm{m}}\left(\sqrt{\frac{T_{\mathrm{m}}}{T_{\mathrm{C}}}}-\sqrt{\frac{T_{\mathrm{m}}}{T_{\mathrm{H}}}}\right) .
$$

This last expression will be used in Section 4.1 to analyze the experimental data on the mass flow rate as a function of the mean rarefaction parameter $\delta_{\mathrm{m}}$.

\section{Experimental mass flow rate.}

The experimental mass flow rates were obtained from the pressure variation rate at $t=0$ in the cold reservoir, according to Eq. (2.2), for various values of the initial pressure and, therefore, for various values of the mean rarefaction parameter, Eq. (2.5).

\subsection{Comparison with numerical results}

The experimental and numerical results of the mass flow rate $\dot{M}$ are compared in Fig. 1 for the two values of the temperature difference $\Delta T$ between the reservoirs. The lines from the numerical simulation, considering the full accommodation at walls and the linear temperature distribution, show good agreements with the plots of the experimental results for the whole range of the experimental conditions, i.e. from the transitional to slip flow regime $0.5<\delta_{\mathrm{m}}<40$. With increase in $\delta_{\mathrm{m}}$, the mass flow rate increases in the transitional flow regime and approaches asymptotically to a constant value in the slip flow regime. The measured results are slightly scattered in the large rarefaction parameter region, which could be caused by very small pressure variations $\left|p_{\infty}-p_{\mathrm{i}}\right|$ in Eq. (2.1) for both the cold and hot reservoirs due to the weak thermal creep flow, resulting in less accurate measurements of the pressure variation rates.

In previous Sections 3.2 and 3.3, it was shown by the numerical simulation that under our experimental conditions the temperature driven mass flow rate can be approximated with $0.2 \%$ accuracy by only the term proportional to the temperature gradient $\dot{M}_{\mathrm{T}}$ as in Eq. (3.5). Therefore, from Eq. (3.5), the remark above and with the help of Eq. (3.2), we can roughly approximate this temperature driven mass flow rate as $\dot{M} \simeq \dot{M}_{\mathrm{T}} \propto d T / d z \sim$ $\Delta T / L$. In Fig. 1, where the experimental mass flow rate is provided, the ratio of the maximums of the vertical axes is set equal to the corresponding $\Delta T$ ratio, namely as $57.9: 37.4$ for two graphs a):b). Thus, it is shown that the plots are quantitatively quite similar in the two graphs, confirming the validity of the approximation. 
Let us now analyze the influence of the gas viscosity on the mass flow rate. Expression (3.7) for the temperature driven mass flow rate as a function of $\delta_{\mathrm{m}}$ is directly proportional to the viscosity coefficient. Furthermore, because the dimensionless mass flow rate $G_{\mathrm{T}}$, is nearly independent of gas species for noble gases (Sharipov \& Bertoldo 2009), then Eq.(3.7) predicts that the mass flow rate is nearly proportional to the viscosity coefficient for a given value of $\delta_{\mathrm{m}}$. More precisely, the viscosity coefficients at the mean temperature $T_{\mathrm{m}}$, calculated from Eq. (2.4), are equal to $2.06,3.29,2.39,2.63$ and $2.40 \times 10^{-5} \mathrm{~Pa} \cdot \mathrm{s}$ for Helium, Neon, Argon, Krypton and Xenon, respectively. As it can be observed in Fig. 1 , the mass flow rates are shown to be nearly proportional to the viscosity coefficients, listed above.

Moreover, it is interesting to note that the mass flow rates for Argon and Xenon show quite similar values, when the mass flow rate $\dot{M}$ is plotted as a function of $\delta_{\mathrm{m}}$, see Fig. 1. Indeed these gas species have similar values of $\mu_{\text {ref }}$ (Argon $2.117 \cdot 10^{-5} \mathrm{~kg} /(\mathrm{s} \cdot \mathrm{m})$ and Xenon $2.107 \cdot 10^{-5} \mathrm{~kg} /(\mathrm{s} \cdot \mathrm{m})$ ) and $\omega$ (Argon 0.81 and Xenon 0.85 ) resulting in similar value not only for $\mu\left(T_{\mathrm{m}}\right)$ but for $\mu$ at every temperature. Consequently, from the previous comments, we can predict for the mass flow rate of these gas species the similar values at the same $\delta_{\mathrm{m}}$, verified in Fig. 1. Let us note that, experimentally, the same $\delta_{\mathrm{m}}$ is obtained for two gases by choosing the respective pressures such as $p_{\mathrm{Ar}} / p_{\mathrm{Xe}}=\sqrt{m_{\mathrm{Xe}} / m_{\mathrm{Ar}}}$, following Eq.(2.5).

\subsection{Influence of gas-surface interaction and temperature profile}

In the numerical simulation, the full accommodation of the molecules at the wall and the linear temperature profile were assumed. However, the incomplete accommodation is often reported even for machined surfaces (Yamaguchi et al. 2012; Hadj Nacer et al. 2014) and the low heat conductivity of the channel's material might result in a non-linear temperature profile (Rojas-Cárdenas et al. 2015). To verify the relevance of these two assumptions, the effects of an incomplete accommodation and of a temperature profile shape, were examined numerically.

From the numerical results, we obtained that the incomplete accommodation influences essentially the mass flow rate. If $\alpha=0.8$, which is close to the value in Rojas-Cárdenas et al. (2015), the mass flow rate increases, compared to its value for the complete accommodation $\alpha=1$, by at most $17.8 \%$ with Helium at the lowest $\delta$. But when $\delta \gtrsim 4$, it decreases compared to its value for $\alpha=1$ and reaches $-7.9 \%$ at $\delta \sim 27.5$. For the other gases, the tendency is similar, but the deviation extent in the mass flow rate compared to its value for $\alpha=1$ is smaller than the case for Helium. It was also found that the mass flow rate decreasing due to the incomplete accommodation is independent of the applied temperature difference.

On the other hand, the exponential temperature profile, which is usually measured for a material with low thermal conductivity, increases the mass flow rate. If we assume the same exponential shape of the temperature distribution as that measured in RojasCárdenas et al. (2015) for the micro tube of the circular cross-section, we found the mass flow rate increasing by at most $6.1 \%$, compared to the linear temperature distribution, with Helium at the lowest $\delta$. For the other gases, the impact of the temperature profile shape on the mass flow rate is much smaller compared to Heluim. In general, the influence of the temperature profile shape is smaller than that of the incomplete accommodation.

The mass flow rates in the case of full accommodation and linear temperature profile were compared in Fig. 2 a) with those with the incomplete accommodation and with the exponential temperature profile, for Argon as a typical example. The same tendency, as observed above in the case of Helium, is clearly seen for Argon on Fig. 2 a): the exponential temperature distribution along the channels' wall increases slightly the mass 

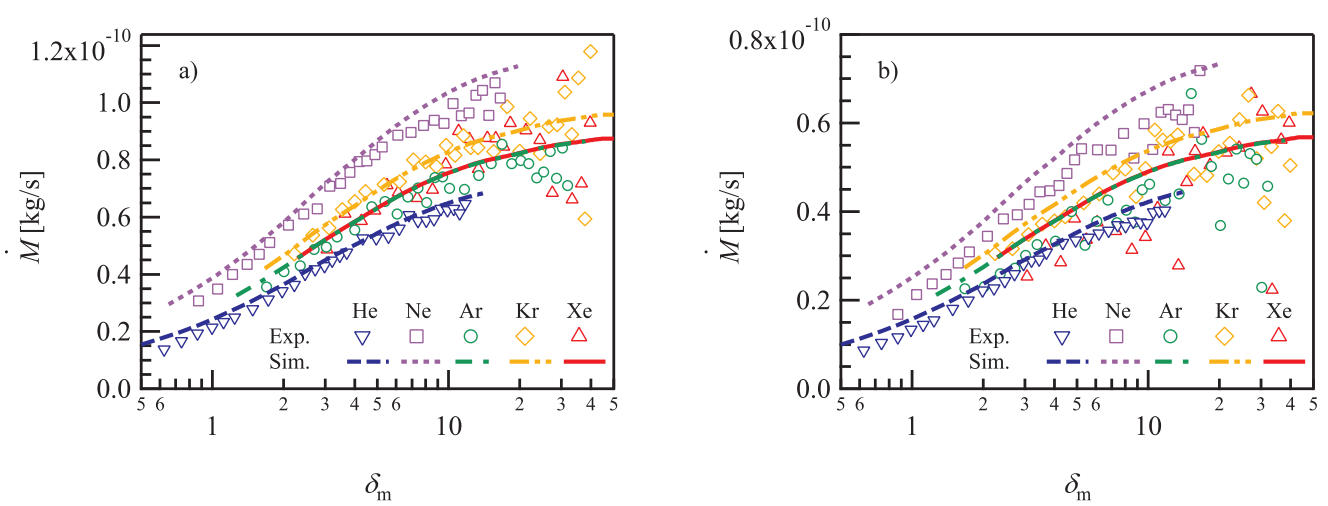

Figure 1. The experimental mass flow rate $\dot{M}$ were compared with the values obtained from the numerical simulations. a) is for $\Delta T=58 \mathrm{~K}$ and b) is for $\Delta T=37 \mathrm{~K}$. The symbols represent the experimental results of $\nabla$ (blue) Helium, $\square$ (purple) Neon, $\bigcirc$ (green) Argon, $\diamond$ (orange) Krypton, and $\triangle$ (red) Xenon, respectively. The results of the simulation considering the full accommodation at walls plotted by a dashed line for Helium, a dotted line for Neon, a dashed-dotted line for Argon, a dashed-two dotted line for Krypton, and a solid line for Xenon, respectively. The maximums of the vertical axes are set in the same ratio as $\Delta T$ for a):b).

flow rate, while the incomplete accommodation decreases it for the higher gas rarefaction (low $\delta$ ) and decreases for high values of $\delta$. To see the tendency above more clearly, the two deviations in the mass flow rate were also plotted in Fig. 2 b). The first deviation $(\dot{M}(\alpha=0.8)-\dot{M}(\alpha=1)) / \dot{M}(\alpha=1)$ shows the influence of the incomplete accommodation of the molecules with the channels' wall, which leads to increase of the mass flow rate compared to the mass flow rate for $\alpha=1$ for the small delta and decrease with the high $\delta$, see Fig. 2 b). The deviation of the mass flow rate due to the change in the shape of the wall temperature profile $\left(\dot{M}\left(T_{\text {exp }}-\dot{M}\left(T_{l i n}\right)\right)\right) / \dot{M}\left(T_{l i n}\right)$ is positive for all $\delta$. As it can be seen from Fig. 2 b), the two effects of the incomplete accommodation and of the temperature profile shape compensate each other in the considered $\delta_{\mathrm{m}}$ range $[3,40]$. Probably, this is a reason why the numerical estimation gives good agreement with the experimental data, see Fig. 1, even though ideal conditions were assumed in the simulations. This agreement could be resulted from the two realistic effects of the incomplete accommodation and non-linear temperature profile varying the mass flow rate in opposite directions.

\subsection{Reduced mass flow rate $G_{\mathrm{T}}$}

Simple explicit expression for the temperature driven mass flow rate is obtained in Section 3.3, Eq.(3.6). However, this expression (3.6) involves the numerical results on $G_{\mathrm{T}}$ coefficient. As it was mentioned above, this coefficient was calculated numerically by various authors. Therefore, we can derive the expression of $G_{\mathrm{T}}$ from our experimental results and compare with the available results of the numerical simulations.

The dimensionless temperature driven mass flow rate $G_{\mathrm{T}}$, see Eq. (3.6), for all gases is plotted in Fig. 3. The numerical results obtained by solving the linearized Boltzmann equation with the Lennard-Jones potential for the flow between parallel plates (Sharipov \& Bertoldo 2009) and the numerical solution of the S-model equation with Hard Sphere potential for the channel with $H / W=0.0367$ cross-section (Graur \& Ho 2014) are plotted in the same Fig. 3. As it is clear from Fig. 3 that the difference in gas species is very small for both the numerical and experimental results. Therefore, the reduced 


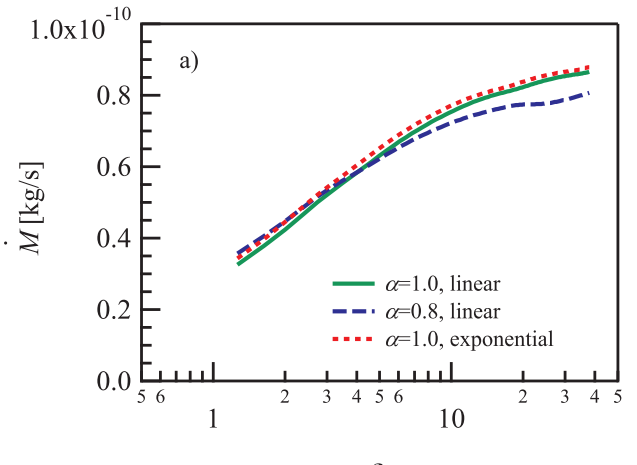

$\delta_{\mathrm{m}}$

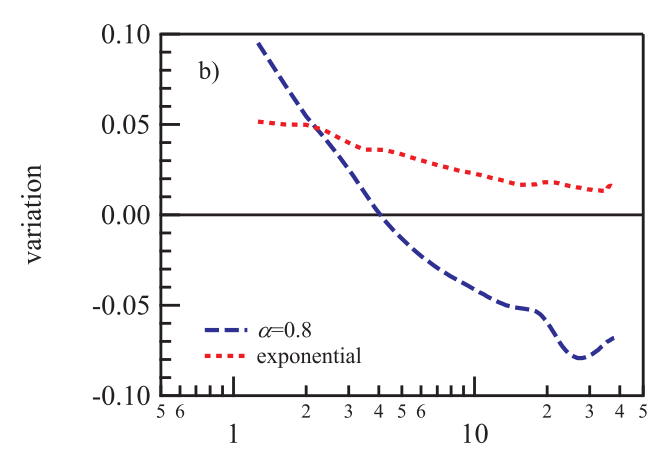

$\delta_{\mathrm{m}}$

FIGURE 2. The effects of the incomplete accommodation and temperature profile are evaluated by the numerical simulations. In a), the mass flow rate of the original full accommodation of the molecules at the wall and the linear temperature profile (green solid line) is plotted with those of the incomplete accommodation and the linear temperature profile (blue dashed line); and of the complete accommodation and the exponential temperature profile (red dotted line); all results for Argon as a typical example. In b), the variations which are the differences for two effects from the original divided by the original mass flow rate are plotted. The vertical axis in (a) is modified from Fig. 1.

flow rate $G_{\mathrm{T}}$ might be considered as independent of gas species at least for noble gases. The reduced flow rates $G_{\mathrm{T}}$ of helium, neon and argon for $\Delta T=58 \mathrm{~K}$ is listed with the measured mass flow rates in Table 1.

Until the slip flow regime, the obtained experimental results show good agreement with the numerical results. However, a small discrepancy appears in the near free molecular regime, i.e. in the small $\delta$ region. It seems very difficult to explain this discrepancy as resulting from an incomplete accommodation at the surface. As it was shown in the numerical simulations (Loyalka 1975; Loyalka \& Hickey 1991; Sharipov \& Seleznev 1998) and through presented here results, an incomplete accommodation would lead to an increase in the reduced flow rate $G_{\mathrm{T}}$ in the small $\delta$ region, and it is not consistent with the results in Fig. 3. Another possibility is the measurement errors increase with decreasing in $\delta$, because the measurements are quite difficult in the free molecular flow regime.

\section{The slip flow regime: the thermal slip coefficient $\sigma_{\mathrm{T}}$}

\subsection{Extraction of the thermal slip coefficient from the mass flow rate}

In the previous section, it was shown that in the transitional and slip flow regimes the measured thermal creep mass flow rate is in very good agreement with the numerical results. However, the mass flow rate can be obtained only numerically through the solution of the kinetic equation. From the previous study (Yamaguchi et al. 2014), it was shown that the thermal creep phenomenon is also important in the slip flow regime. Therefore, it will be worth to have an analytical solution in the slip flow regime (Rojas-Cárdenas et al. 2015).

The flow in the micro channel at the slip flow regime could be described by the Stokes equation. As mentioned above, the pressure profile along the channel could be negligible, and the thermal creep flow could be assumed as a stationary flow at $t=0$ where the 


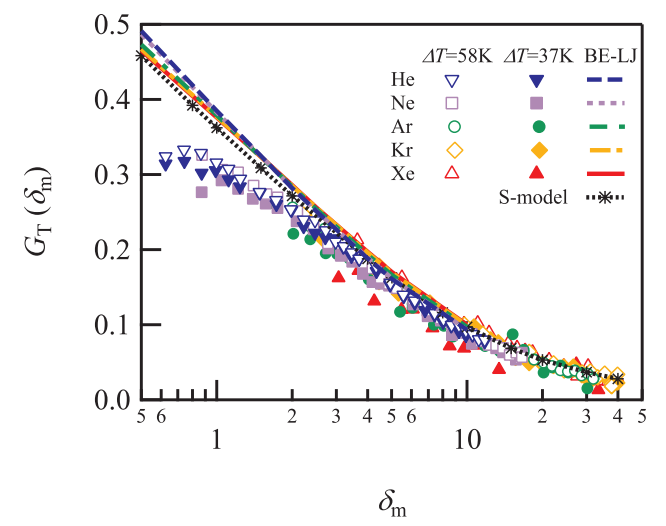

FIGURE 3 . The reduced flow rate $G_{\mathrm{T}}$ obtained from the experimental results are plotted for both $\Delta T=58 \mathrm{~K}$ and $\Delta T=37 \mathrm{~K}$. The symbols represent the experimental results for $\Delta T=58 \mathrm{~K}$ of $\nabla$ Helium, $\square$ Neon, $\bigcirc$ Argon, $\diamond$ Krypton, and $\Delta$ Xenon, respectively. The data for $\Delta T=37 \mathrm{~K}$ are plotted with filled markers. The numerical results for the rectangular channel $(H / W=0.0367)$ for the full accommodation, obtained by the S-model kinetic simulation in (Graur \& Ho 2014), are plotted by a dotted line with markers. The numerical results for the flow between two parallel plates with the full accommodation and for Lennard-Jones intermolecular potential in (Sharipov \& Bertoldo 2009) for $\delta_{\mathrm{m}}<10$ are also plotted by a dashed line for Helium, a dotted line for Neon, a dashed-dotted line for Argon, a dashed-two dotted line for Krypton, and a solid line for Xenon, respectively.

mass flow rate is calculated; thus, the equation has the form

$$
\frac{\partial^{2} u_{z}}{\partial x^{2}}+\frac{\partial^{2} u_{z}}{\partial y^{2}}=0
$$

where $z$-axis coincides with the direction of the temperature gradient and the thermal creep flow is induced in the $z$ direction with the velocity $u_{z}$. The thermal slip boundary condition on a flat wall has the form

$$
\left.u_{z}\right|_{\mathrm{wall}}=\sigma_{T} \frac{\mu}{\rho T} \frac{d T}{d z},
$$

where $\rho$ is the gas density. The solution of the Stokes equation, Eq. (5.1), with the thermal slip boundary condition, Eq. (5.2), is a constant velocity over the whole cross section (Sharipov 2011). Thus, the mass flow rate is obtained as,

$$
\dot{M}=\sigma_{T} H W \frac{\mu}{T} \frac{d T}{d z} .
$$

By using Eq. (2.4) for the viscosity coefficient, the mass flow rate is expressed as,

$$
\dot{M}=\sigma_{T} \frac{H W \mu_{\text {ref }}}{T_{\text {ref }}^{\omega}} \mathcal{F},
$$

where $\mathcal{F}=T^{\omega-1} d T / d z$, and only $\mathcal{F}$ varies by local conditions. As it was pointed out in Section 4.1, in this study the temperature profile along the channel was not measured; therefore, the mean value of $\mathcal{F}$ was calculated by integrating its expression along the channel:

$$
\overline{\mathcal{F}}=\frac{1}{L} \int_{T_{\mathrm{C}}}^{T_{\mathrm{H}}} T^{\omega-1} \mathrm{dT}=\frac{T_{\mathrm{H}}^{\omega}-T_{\mathrm{C}}^{\omega}}{\omega L},
$$

so we do not need to do any assumption on the temperature distribution shape. 
TABLE 1 . The measured mass flow rates $\dot{M}\left[\times 10^{-10} \mathrm{~kg} / \mathrm{s}\right]$ and reduced flow rates $G_{\mathrm{T}}$ of typical gas species for $\Delta T=58 \mathrm{~K}$.

\begin{tabular}{|c|c|c|c|c|c|c|c|c|}
\hline$\delta_{\mathrm{m}}$ & $\begin{array}{c}\mathrm{He} \\
\dot{M}\end{array}$ & $G_{\mathrm{T}}$ & $\delta_{\mathrm{m}}$ & $\begin{array}{l}\mathrm{Ne} \\
\dot{M}\end{array}$ & $G_{\mathrm{T}}$ & $\delta_{\mathrm{m}}$ & $\begin{array}{l}\mathrm{Ar} \\
\dot{M}\end{array}$ & $G_{\mathrm{T}}$ \\
\hline 0.624 & 0.137 & 0.324 & 0.873 & 0.307 & 0.326 & 1.70 & 0.355 & 0.264 \\
\hline 0.739 & 0.167 & 0.333 & 1.05 & 0.349 & 0.306 & 2.01 & 0.409 & 0.256 \\
\hline 0.865 & 0.193 & 0.328 & 1.22 & 0.399 & 0.303 & 2.36 & 0.430 & 0.231 \\
\hline 0.998 & 0.214 & 0.316 & 1.41 & 0.435 & 0.285 & 2.70 & 0.487 & 0.228 \\
\hline 1.11 & 0.233 & 0.308 & 1.58 & 0.469 & 0.274 & 3.03 & 0.496 & 0.206 \\
\hline 1.24 & 0.249 & 0.294 & 1.75 & 0.511 & 0.270 & 3.38 & 0.531 & 0.199 \\
\hline 1.48 & 0.278 & 0.276 & 2.10 & 0.571 & 0.251 & 4.01 & 0.554 & 0.175 \\
\hline 1.73 & 0.313 & 0.266 & 2.44 & 0.611 & 0.232 & 4.73 & 0.636 & 0.169 \\
\hline 1.98 & 0.341 & 0.253 & 2.78 & 0.629 & 0.209 & 5.37 & 0.655 & 0.153 \\
\hline 2.22 & 0.363 & 0.239 & 3.14 & 0.708 & 0.208 & 6.04 & 0.611 & 0.127 \\
\hline 2.47 & 0.402 & 0.239 & 3.48 & 0.718 & 0.190 & 6.72 & 0.670 & 0.125 \\
\hline 2.72 & 0.418 & 0.225 & 3.83 & 0.756 & 0.182 & 7.41 & 0.702 & 0.119 \\
\hline 2.98 & 0.429 & 0.210 & 4.18 & 0.795 & 0.175 & 8.08 & 0.651 & 0.102 \\
\hline 3.22 & 0.448 & 0.204 & 4.52 & 0.796 & 0.162 & 8.75 & 0.739 & 0.106 \\
\hline 3.47 & 0.465 & 0.196 & 4.87 & 0.819 & 0.154 & 9.41 & 0.741 & 0.0989 \\
\hline 3.71 & 0.476 & 0.188 & 5.24 & 0.845 & 0.148 & 10.1 & 0.700 & 0.0874 \\
\hline 4.33 & 0.526 & 0.178 & 6.09 & 0.886 & 0.134 & 11.7 & 0.697 & 0.0751 \\
\hline 4.94 & 0.526 & 0.156 & 6.94 & 0.896 & 0.119 & 13.4 & 0.746 & 0.0698 \\
\hline 5.57 & 0.532 & 0.140 & 7.83 & 0.920 & 0.108 & 15.1 & 0.787 & 0.0654 \\
\hline 6.19 & 0.560 & 0.132 & 8.70 & 0.939 & 0.099 & 16.8 & 0.855 & 0.0638 \\
\hline 6.80 & 0.607 & 0.130 & 9.55 & 0.928 & 0.089 & 18.5 & 0.787 & 0.0533 \\
\hline 7.41 & 0.587 & 0.115 & 10.4 & 0.997 & 0.087 & 20.2 & 0.798 & 0.0496 \\
\hline 8.04 & 0.587 & 0.107 & 11.3 & 0.954 & 0.077 & 21.8 & 0.787 & 0.0453 \\
\hline 8.67 & 0.592 & 0.0996 & 12.2 & 0.965 & 0.072 & 23.6 & 0.737 & 0.0393 \\
\hline 9.28 & 0.629 & 0.0987 & 13.1 & 1.03 & 0.072 & 25.2 & 0.758 & 0.0377 \\
\hline 9.90 & 0.624 & 0.0918 & 13.9 & 1.04 & 0.069 & 26.9 & 0.829 & 0.0388 \\
\hline 10.5 & 0.629 & 0.0871 & 14.8 & 0.956 & 0.059 & 28.6 & 0.736 & 0.0323 \\
\hline 11.1 & 0.613 & 0.0803 & 15.7 & 1.07 & 0.062 & 30.3 & 0.842 & 0.0349 \\
\hline 11.7 & 0.646 & 0.0800 & 16.5 & 1.02 & 0.056 & 31.9 & 0.710 & 0.0279 \\
\hline
\end{tabular}

The thermal slip coefficient $\sigma_{\mathrm{T}}$ was calculated from the measured mass flow rate by using the analytical expression for the thermal creep mass flow rate, Eqs. (5.4) and (5.5):

$$
\sigma_{\mathrm{T}}=\dot{M} / \dot{M}_{\mathrm{ref}}, \quad \dot{M}_{\mathrm{ref}}=\frac{H W \mu_{\mathrm{ref}}}{T_{\mathrm{ref}}^{\omega}} \frac{T_{\mathrm{H}}^{\omega}-T_{\mathrm{C}}^{\omega}}{\omega L} .
$$

The calculated thermal slip coefficient $\sigma_{\mathrm{T}}$, obtained in the slip flow regime, $\delta_{\mathrm{m}}=[10,40]$, was plotted in Fig. 4 . To validate the independence of the coefficient $\sigma_{\mathrm{T}}$ from $\delta_{\mathrm{m}}$, they are plotted against $\delta_{\mathrm{m}}$. The plots are a little scattered resulting from the scattered results of the mass flow rate discussed in Section 4.1, but the value seems to be independent of the rarefaction parameter $\delta_{\mathrm{m}}$ and of the temperature difference $\Delta T$ as in RojasCárdenas et al. (2015). Therefore, the obtained results are averaged over both of the two temperature differences and they are listed in Table 2 as "all" and plotted with horizontal lines in Fig. 4. The 95\% confidence interval for the averaging was listed as errors in Table 2. To see the effect of the temperature difference, the averaged values for each $\Delta T$ cases are also listed in Table 2. The differences among "all", $\Delta T=58 \mathrm{~K}$ and $\Delta T=37 \mathrm{~K}$ are 


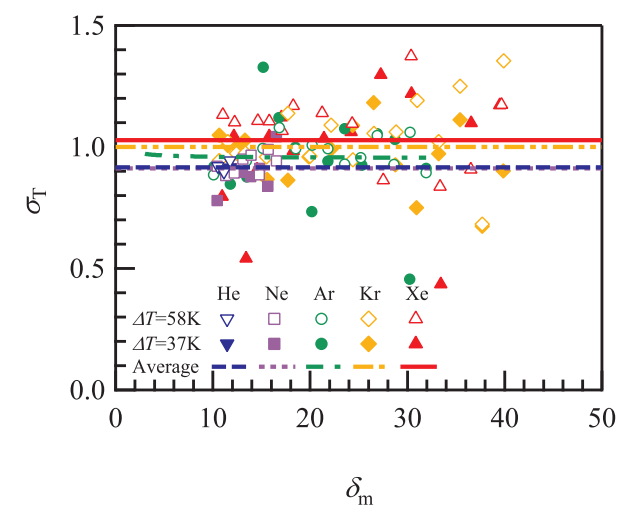

FIGURE 4 . The thermal slip coefficients $\sigma_{\mathrm{T}}$ obtained from the experimental results are plotted for $\Delta T=58 \mathrm{~K}$ and $\Delta T=37 \mathrm{~K}$. They are plotted against $\delta_{\mathrm{m}}$, validating that the coefficient is independent of $\delta_{\mathrm{m}}$. The symbols represent the experimental results for $\Delta T=58 \mathrm{~K}$ of $\nabla$ Helium, $\square$ Neon, $\bigcirc$ Argon, $\diamond$ Krypton, and $\triangle$ Xenon, respectively. The data for $\Delta T=37 \mathrm{~K}$ are plotted with filled markers. The averaged values over both two $\Delta T$ cases are plotted by a dashed line for Helium, a dotted line for Neon, a dashed-dotted line for Argon, a dashed-two dotted line for Krypton, and a solid line for Xenon, respectively.

TABLE 2. Obtained thermal slip coefficients from the averaged mass flow rate over the range of $\delta_{\mathrm{m}}=[10,40]$. The errors are $95 \%$ confidence interval of the fitting procedure.

\begin{tabular}{cccc}
\hline & all & $\Delta T=58 \mathrm{~K}$ & $\Delta T=37 \mathrm{~K}$ \\
\hline $\mathrm{He}$ & $0.916 \pm 0.018$ & $0.921 \pm 0.171$ & $0.911 \pm 0.030$ \\
$\mathrm{Ne}$ & $0.912 \pm 0.033$ & $0.929 \pm 0.035$ & $0.895 \pm 0.065$ \\
$\mathrm{Ar}$ & $0.957 \pm 0.057$ & $0.971 \pm 0.038$ & $0.944 \pm 0.116$ \\
$\mathrm{Kr}$ & $1.000 \pm 0.052$ & $1.039 \pm 0.082$ & $0.961 \pm 0.070$ \\
$\mathrm{Xe}$ & $1.029 \pm 0.078$ & $1.074 \pm 0.082$ & $0.983 \pm 0.142$
\end{tabular}

very small and within the errors interval. It is important to note that the number of the data in the range, used for the averaging, was different for every gas species, since the rarefaction parameter $\delta$, Eq. (2.3), is different between gas species at the same pressure condition due to the difference in the molecular weights.

\subsection{Extraction of $\sigma_{\mathrm{T}}$ by asymptotic expression for the mass flow rate}

The above mentioned mass flow rate, obtained by solving the Stokes equation with thermal slip boundary condition, is a first order solution. The higher order solution for the dimensionless temperature driven mass flow rate $G_{\mathrm{T}}$ was obtained from the kinetic theory in form of an asymptotic expression in Loyalka \& Hickey (1989) and in term of the dimensional mass flow rate, it reads

$$
\dot{M}=\frac{H W \mu}{T} \frac{d T}{d z}\left[A^{\mathrm{T}}+\frac{B^{\mathrm{T}}}{\delta}+O\left(\frac{1}{\delta^{2}}\right)\right],
$$

where the coefficients $A^{\mathrm{T}}$ and $B^{\mathrm{T}}$ as obtained by Loyalka \& Hickey (1989) are $A^{\mathrm{T}}=$ 0.9924 and $B^{\mathrm{T}}=-1.3284$. It is to add that here, as previously, the pressure gradient is assumed to be small and only the term with the temperature gradient is retained. By 
comparing Eqs. (5.3) and (5.7), it is clear that the both expressions are similar and $A^{\mathrm{T}}$ can be identified to the thermal slip coefficient $\sigma_{\mathrm{T}}$ in the slip flow regime. However, the asymptotic expression, Eq.(5.7), contains the additional term of the order of $O(1 / \delta)$ and therefore allows to determine $\sigma_{T}$ coefficient more accurately. As in the case with the first order extraction, expression (5.7) was integrated along the channel by using the mass conservation property. To integrate expression (5.7), the rarefaction parameter $\delta$ was also related to the mean rarefaction parameter $\delta_{\mathrm{m}}$ and the local temperature and pressure values, see Rojas-Cárdenas et al. (2015) for more details. Finally, the expression of the mass flow rate through mean and reservoirs' values is obtained

$$
\begin{aligned}
\dot{M} & =\dot{M}_{\text {ref }}\left[A^{\mathrm{T}}+\frac{B^{\mathrm{T}} B_{2}}{\delta_{\mathrm{m}}}+O\left(\frac{1}{\delta_{\mathrm{m}}^{2}}\right)\right], \\
B_{2} & =\frac{T_{\mathrm{H}}^{2 \omega+1 / 2}-T_{\mathrm{C}}^{2 \omega+1 / 2}}{(2 \omega+1 / 2) T_{\mathrm{m}}^{\omega+1 / 2}} \frac{\omega}{T_{\mathrm{H}}^{\omega}-T_{\mathrm{C}}^{\omega}} .
\end{aligned}
$$

The previous expression, by analogy with Eq. (5.6), can be rewritten in the following form

$$
\mathcal{G}\left(\delta_{\mathrm{m}}\right)=\dot{M} / \dot{M}_{\mathrm{ref}}=A^{\mathrm{F}}+\frac{B^{\mathrm{F}} B_{2}}{\delta_{\mathrm{m}}} .
$$

It is clear that the measured mass flow rate can be fitted in form of Eq. (5.9) and the fitting coefficient $A^{\mathrm{F}}$ can be associated with the coefficient $\sigma_{\mathrm{T}}$.

Since the asymptotic expression allowed to enlarge the applicable flow regime to the transitional flow regime, the measured mass flow rate in the range of $\delta_{\mathrm{m}}=[3,40]$ was employed for the fitting procedure. The experimental data with the fitting curves are plotted in Fig. 5. The coefficients $A^{\mathrm{F}}$ and $B^{\mathrm{F}}$, obtained from the fitting of the experimental data according to Eq. (5.9) are listed in Table 3. The coefficient $B_{2}$ was quite close to unity and the largest deviation from unity was less than $0.1 \%$. Therefore, to calculate the thermal slip coefficients from all the data for both $\Delta T=58 \mathrm{~K}$ and $37 \mathrm{~K}$, $B_{2}=1$ was assumed. The errors from $B_{2}$ in the calculation of the coefficient $B^{\mathrm{F}}$ was also neglected, since the values were almost constant and the errors in the temperature measurement should be very small.

These results are theoretically much reliable compared to the first order extraction in Table 2, because a greater number of data were employed for the fitting procedure, which is especially true for Helium. In addition, the values are quantitatively in quite good agreement with the numerical (Sharipov \& Bertoldo 2009) and the experimental results in the literature (Annis 1972; Porodnov et al. 1978; Rojas-Cárdenas et al. 2015). This agreement indicates notably that the thermal slip coefficient is independent of the cross-sectional shape of the channel.

Comparing between gas species, the thermal slip coefficient increases with an increase in the molecular weight, except Neon. Neon gives a little smaller value compared with Helium. It shows the same tendency with the case of the first order extraction, see Table 2. This tendency is exactly the same with the numerical results (Sharipov 2011) and with the experimental values obtained previously in Annis (1972); Porodnov et al. (1978).

As it is clear from Table 3 , the $B^{\mathrm{F}}$ coefficients are also close to the $\overline{B^{\mathrm{T}}}=-1.3284$, obtained numerically in Loyalka \& Hickey (1989) under the complete accommodation assumption. The comparison between the experimentally obtained coefficients and the numerical data, especially for the coefficient $B^{\mathrm{T}}$, which is more sensitive to the accommodation coefficient (Loyalka \& Hickey 1989), indicates that the experimental accommodation coefficient is not so far from unity. 


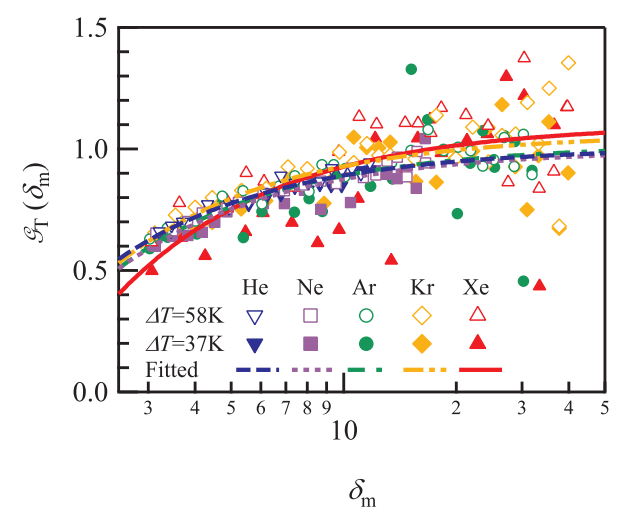

FIGURE 5. Function $\mathcal{G}\left(\delta_{\mathrm{m}}\right)$, Eq. (5.9), as a function of $\delta_{\mathrm{m}}$, obtained from the experimental results are plotted for $\Delta T=58 \mathrm{~K}$ and $\Delta T=37 \mathrm{~K}$. The symbols represent the experimental results for $\Delta T=58 \mathrm{~K}$ of $\nabla$ Helium, $\square$ Neon, $\bigcirc$ Argon, $\diamond$ Krypton, and $\Delta$ Xenon, respectively. The data for $\Delta T=37 \mathrm{~K}$ are plotted with filled markers. The fitting curves over both two $\Delta T$ cases are plotted by a dashed line for Helium, a dotted line for Neon, a dashed-dotted line for Argon, a dashed-two dotted line for Krypton, and a solid line for Xenon, respectively.

TABLE 3. Obtained thermal slip coefficients $A^{\mathrm{F}}$ and $B^{\mathrm{F}}$ by the fitting of the asymptotic mass flow rate expression over the range of $\delta_{\mathrm{m}}=[3,40]$. $B_{2}$ was assumed to be unity in the calculation for "all". The errors are $95 \%$ confidence interval of the fitting procedure, and uncertainties from $B_{2}$ are neglected.

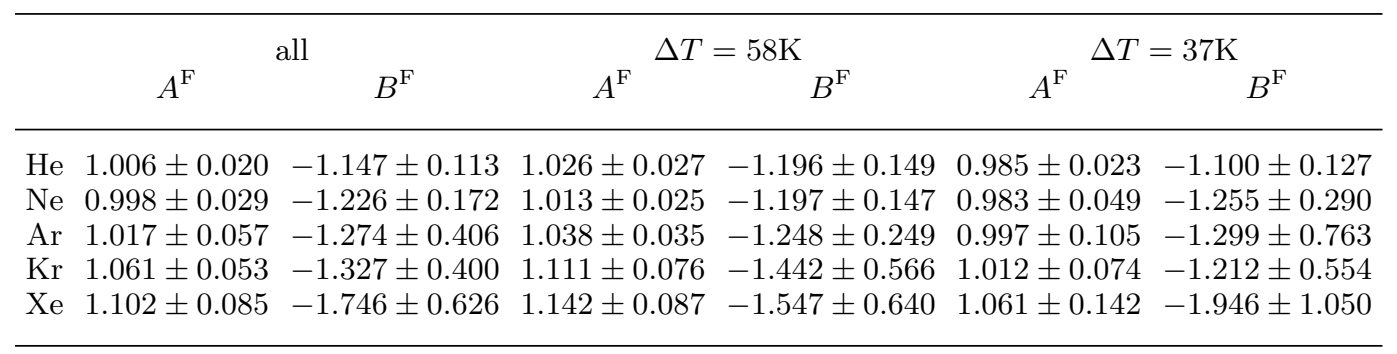

It would be also interesting to calculate the accommodation coefficient from the obtained experimentally thermal slip coefficient, by using, for example, the following expression proposed in (Sharipov 2011) in the frame of the Maxwell diffuse-specular boundary condition and using the S-model kinetic equation

$$
\sigma_{\mathrm{T}}=0.75(1+0.5 \alpha) .
$$

However, the thermal slip coefficient is very sensitive to the type of kinetic equation (Smodel kinetic equation or Boltzmann equation), used for its numerical calculation, and especially, to the implemented intermolecular potential (Hard-Sphere model, Maxwellian molecules, Lenard-Jones potential). In the case of full accommodation at a surface, the values of $\sigma_{\mathrm{T}}$ are dispersed for different potentials within $16 \%$, as stated in Sharipov (2011). The same problem appears when trying to simulate diffuse-specular scattering: the disagreement between the results obtained by the variational method applied to the S-model with diffuse-specular boundary conditions and the solution of the Boltzmann 
equation with the HS potential varies from $3 \%$ for $\alpha=0$ to $12 \%$ for $\alpha=1$ (Sharipov 2011).

Moreover, when one extracts an experimental value of the accommodation coefficient from the thermal slip coefficient by using Eq. (5.10), it is clear that the uncertainties on $\alpha$ represents $8 / 3$ of the uncertainties on $\sigma_{\mathrm{T}}$ and so would lie beyond the acceptable accuracy, especially for Argon and Xenon. In addition, taking into account this large difference in numerical values of $\sigma_{\mathrm{T}}$ described above, the implementation of Eq. (5.10) itself to extract the value of the accommodation coefficient can engender a large additional error. Therefore, it is not reasonable to calculate the accommodation coefficient from the experimental results in this study. Nonetheless, the accommodation coefficient which corresponds to the obtained thermal slip coefficient would be not so far from unity, which is in consistence with the above mentioned characteristics from the $B^{\mathrm{T}}$ coefficient.

\section{Conclusion}

The thermal slip coefficient in the slip flow regime and the reduced flow rate in the transitional flow regime were obtained for five noble gases in the rectangular cross section geometry from the experimental measurement of a pure thermal creep flow.

The mass flow rate was compared with the numerical result by solving the S-model kinetic equations, showing good agreement for all cases in all flow conditions. Then, the numerical simulation was employed to validate the assumptions for involved methodology.

The reduced flow rate $G_{\mathrm{T}}$ was directly calculated from the experimentally measured mass flow rate by assuming the negligible variation in pressure and the rarefaction parameter $\delta$ in the micro channel. The obtained values showed good agreement with the numerical results.

The thermal slip coefficient $\sigma_{\mathrm{T}}$ of five noble gases was affected by gas species and it increases with increasing in the molecular weight, except Neon. Neon showed slightly smaller value than the result of Helium. Analyzing the influence of the channel geometry, it was found that the $\sigma_{\mathrm{T}}$ coefficient of the rectangular channel showed quite close value to that of the circular tube, indicating the value is independent of the cross-sectional shape of the channel.

\section{Acknowledgments}

The micro channel and the blocks were fabricated at Institute for Micro Process Engineering (IMVT), Karlsruhe Institute of Technology (KIT) in the framework of the European Community's Seventh Framework Program (FP7/2007-203 under grant agreement n 215504). This study was partially supported by the Overseas Visits Program for Young Researchers by Nagoya University Venture Business Laboratory and the program "Creation of Materials, Processes and Systems Provided with New and High Functionality" in the fund "Institutional Program for Young Researcher Overseas Visit" sponsored by JSPS. This work has been carried out in the framework of the Labex MEC (ANR10-LABX-0092) and of the A*MIDEX project (ANR-11-IDEX-0001-02), funded by the "Investissements d'Avenir" French Government program managed by the French National Research Agency (ANR).

\section{REFERENCES}

Alexeenko, A.A., Gimelshein, S.F., Muntz, E.P. \& Ketsdever, A.D. 2006 Kinetic mod- 
eling of temperature driven flows in short microchannels. International Journal of Thermal Sciences 45 (11), 1045-1051.

Annis, B. K. 1972 Thermal creep in gases. Journal of Chemical Physics 57 (7), 2898-2905.

BIRD, G. A. 1994 Molecular Gas Dynamics and the Direct Simulation of Gas Flows. Oxford Science Publications, Oxford University Press Inc., New York.

Cercignani, C. 1990 Mathematical methods in kinetic theory. Plenum Press New York Press, New York, London.

Dongari, N. \& Agrawal, A. 2012 Modeling of navier-stokes equations for high knudsen number gas flows. International Journal of Heat and Mass Transfer 55, 4352-4358.

Graur, I. \& Ho, M. T. 2014 Rarefied gas flow through a long rectangular channel of variable cross section. Vacuum 101, 328-332.

Gupta, N.K. \& GiAnchandani, Y.B. 2008 Thermal transpiration in zeolites: A mechanism for motionless gas pumps. Applied Physics Letters 93, 193511.

Gupta, Naveen K, An, Seungdo \& Gianchandani, Yogesh B 2012 A si-micromachined 48stage knudsen pump for on-chip vacuum. Journal of Micromechanics and Microengineering $22(10), 105026$.

Hadj Nacer, M., Graur, I., Perrier, P., Méolans, J. G. \& Wuest, M. 2014 Gas flow through microtubes with different internal surface coatings. Journal of Vacuum Science and Technology A 32, 021601.

Han, Y., Muntz, E. P., Alexeenko, A. \& Young, M. 2007 Experimental and Computational Studies of Temperature Gradient-Driven Molecular Transport in Gas Flows through Nano/Microscale Channels. Nanoscale and Microscale Thermophysical Engineering 11 (1), $151-175$.

Kogan, M. N. 1969 Rarefied gas dynamics. Plenum Press New York.

LOYALKA, S. K. 1975 Kinetic theory of thermal transpiration and mechanocaloric effects. ii. Journal of Chemical Physics 63 (9), 4054-4060.

LoyAlKA, S. K. \& HiCKEY, K. A. 1989 Plane poiseuille flow: Near continuum results for a rigid sphere gas. Physica A 160, 395-408.

LOYALKA, S. K. \& HiCKeY, K. A. 1991 Kinetic theory of thermal transpiration and the mechanocaloric effect: Planar flow of a rigid sphere gas with arbitrary accommodation at the surface. Journal of Vacuum Science and Technology A 9, 158-163.

Martini, V, Bernardini, S, Bendahan, M, Aguir, K, Perrier, P \& Graur, I 2012 Microfluidic gas sensor with integrated pumping system. Sensors and Actuators B: Chemical 170, 45-50.

Ohwada, T., Sone, Y. \& Aoki, K. 1989 Numerical analysis of the shear and thermal creep flows of a rarefied gas over a plane wall on the basis of the linearized Boltzmann equation for hard-sphere molecules. Physics of Fluids A 1 (9), 1588-1599.

Passian, A., Warmack, RJ, Ferrell, TL \& Thundat, T. 2003 Thermal transpiration at the microscale: a Crookes cantilever. Physical review letters 90 (12), 124503.

Porodnov, B., Kulev, A. N. \& Tuchvetov, F. T. 1978 Thermal transpiration in a circular capillary with a small temperature difference. Journal of Fluid Mechanics 88 (4), 609-622.

Rojas-Cárdenas, M., Graur, I., Perrier, P. \& MÉolans, J. G. 2011 Thermal transpiration flow: A circular cross-section microtube submitted to a temperature gradient. Physics of Fluids 23, 031702.

Rojas-Cárdenas, M., Graur, I., Perrier, P. \& Méolans, J. G. 2013 Time-dependent experimental analysis of a thermal transpiration rarefied gas flow. Physics of Fluids 25, 072001.

Rojas-Cárdenas, M., Graur, I., Perrier, P. \& Méolans, J. G. 2015 A new method to measure the thermal slip coefficient. International Journal of Heat and Mass Transfer $\mathbf{8 8}$, 766-774.

ShARIPOV, F. 1999 Non-isothermal gas flow through rectangular microchannels. Journal of Micromechics and Microengineering 9 (4), 394-401.

SharIPov, F. 2011 Data on the Velocity Slip and Temperature Jump on a Gas-Solid interface. Journal of Physical and Chemical Reference Data 40 (2), 023101.

Sharipov, F. \& Bertoldo, G. 2009 Poiseuille flow and thermal creep based on the Boltzmann equation with the Lennard-Jones potential over a wide range of the Knudsen number. Physics of Fluids 21, 067101. 
Mass Flow Rate Measurementof Thermal Creep Flowfrom Transitional to Slip Flow Reginße

Sharipov, F. \& Seleznev, V. 1998 Data on internal rarefied gas flows. Journal of Physical and Chemical Reference Data 27 (6), 657-706.

Sone, Y. 2002 Kinetic Theory and Fluid Mechanics. Birkhäuser, Boston.

Stvorik, T. S., PARK, H. S. \& LOYALKA, S. K. 1978 Thermal transpiration: A comparison of experiment and theory. Journal of Vacuum Science Technology 15 (6), 1844-1852.

TAkAishi, T. \& Sensui, Y. 1963 Thermal transpiration effect of hydrogen, raregases and methane,. Transactions of the Faraday Society 59, 2503-2514.

VArgo, SE, Muntz, EP, Shiflett, GR \& TANG, WC 1999 Knudsen compressor as a microand macroscale vacuum pump without moving parts or fluids. Journal of Vacuum Science \& Technology A: Vacuum, Surfaces, and Films 17, 2308.

YamAGUChi, H., MatsudA, Y. \& NiImi, T. 2012 Tangential momentum accommodation coefficient measurements for various materials and gas species. Journal of Physics: Conference Series 362, 012035.

Yamaguchi, H., Rojas-Cárdenas, M., Perrier, P., Graur, I. \& Nimmi, T. 2014 Thermal transpiration flow through a single rectangular channel. Journal of Fluid Mechanics $\mathbf{7 4 4}$, 169-182.

Young, M., Han, Y.L., Muntz, EP, Shiflett, G., Ketsdever, A. \& Green, A. 2003 Thermal transpiration in microsphere membranes. In AIP Conference Proceedings, pp. 743-751. iop institute of physics publishing ltd. 